\title{
Friedensförderung und Konflikttransformation - Notwendigkeiten eines stärkeren deutschen Engagements
}

\author{
Edelgard Bulmahn*
}

I.

Festrede bei der Verleihung der Abschlussurkunden im Studiengang Master of Peace and Security Studies (MPS) am Institut für Friedensforschung und Sicherheitspolitik an der Universität Hamburg (IFSH) am 09. Oktober 2012 in Hamburg.

$\mathrm{D}$ as IFSH leistet seit nunmehr 40 Jahren wichtige, ja unverzichtbare Beiträge für zivile Krisenprävention und Konfliktlösung. Ich bin aber auch deshalb sehr gerne nach Hamburg gekommen, weil der Studiengang des "Master of Peace and Security Studies" eng mit der Deutschen Stiftung Friedensforschung (DSF) verbunden ist, die ich 2001 als Bundesministerin unter tatkräftiger Mithilfe des damaligen Direktors des ISFH, dem leider viel zu früh verstorbenen Dieter Lutz, ins Leben gerufen habe.

Der Studiengang ist ein gelungenes Beispiel für eine ertragreiche Kooperation zwischen einer Universität und einer Forschungseinrichtung. Diese schafft den Rahmen, Kompetenzen und Forschungsmöglichkeiten miteinander zu verbinden. Und sie sichert die Ausbildung des wissenschaftlichen Nachwuchses in einem Wissenschaftsfeld, das im universitären Rahmen so nicht möglich wäre.

Die 26 Absolventinnen und Absolventen des Master-Programms haben sich in den vergangenen Semestern Wissen, Fähigkeiten und Handlungskompetenz angeeignet, die sie in besonderer Weise befähigen für eine Tätigkeit in Wissenschaft und Forschung, in der Politikberatung, aber auch für ganz konkrete Einsätze in Friedensmissionen oder Projekten der Entwicklungszusammenarbeit. Internationalität und Disziplin übergreifende Arbeit sind für sie keine bloßen Schlagworte.

\section{II.}

Frieden ist der höchste Wert, nach dem wir streben können. Ein Frieden, der immer wieder bedroht wird, den man nicht geschenkt bekommt und der täglich neu errungen und gesichert werden muss. Albert Einstein beschreibt Frieden so: Frieden sei kein gegebener Zustand, sondern Ziel und Ergebnis gesellschaftlicher und politischer Gestaltung. Frieden ist ein Prozess, der vorausschauend und dauerhaft gestaltet werden will. Frieden verlangt klare Vorstellungen und einen Konsens darüber, wie wir miteinander leben wollen.

Edelgard Bulmahn, MdB, Bundesministerin a. D., Mitglied des Auswärtigen Ausschusses des Deutschen Bundestages.
Die Abwesenheit von Krieg ist deshalb noch lange kein Garant für die Überwindung der Konflikte und Unsicherheiten in der Welt. Konflikte und gewalttätige Auseinandersetzungen haben gerade in den vergangenen Jahren ihr Gesicht immer wieder verändert. Die Welt erlebt Umbrüche und lässt neue Krisenherde und Problemsituationen entstehen, die sich in Kriegen und anderen Formen gewaltsamer Konfliktaustragungen entladen. Militärische Mittel, das zeigt sich allerorten, sind immer weniger in der Lage, die Konflikte zu befrieden. Und vor allem tragen sie nur selten dazu bei, den Ausbruch offener Feindseligkeiten zu unterbinden.

Seit dem Ende des „Kalten Krieges“ haben sich die Ursachen gewaltsamer Konflikte stark verändert. Waren Krieg und militärische Auseinandersetzungen in der Vergangenheit geprägt durch Grenzkonflikte, Hegemonialansprüche, widerstreitende Wirtschaftsinteressen oder ethnisch und religiös aufgeheizte Konflikte, so sind heute weitere Ursachen hinzugekommen: Staatszerfall, die Entstaatlichung von Gewalt durch asymmetrische Konflikte, Genozid und Massenvertreibungen, Terrorismus, organisierte Kriminalität, Hunger, Migration und Verteilungskonflikte um Ressourcen. Dass sich im Grunde alle diese Themen mehr oder minder in Ihren Masterarbeiten wiederfinden, zeigt, welche Bedeutung sie haben und wie wichtig es ist, sich mit ihnen auseinanderzusetzen.

Diese neuen Konfliktursachen und -formen überfordern althergebrachte Lösungsansätze. Friedensforschung ist deshalb heute wichtiger denn je. Wenn wir Ursachen und Verläufe von aktuellen wie auch vergangenen Konflikten besser kennen und die handelnden Personen und ihre Motivation besser verstehen, dann können wir daraus Friedensstrategien entwickeln und friedensbildende Prozesse in Gang setzen und begleiten. Wichtigste Aufgabe der Friedensforschung ist es daher, einen Beitrag dazu zu leisten, dass Konflikte präventiv bearbeitet werden können, dass Politik und Öffentlichkeit frühzeitig auf Gefahren hingewiesen werden, deeskalierende bzw. friedenssichernde Maßnahmen ergriffen werden können. Friedensforschung hat aber nicht nur frühzeitig auf Gefahren hinzuweisen, sondern sie sollte auch politische Entscheidungen kritisch auf mögliche Konsequenzen hin analysieren und gegebenenfalls Alternativen aufzeigen.

Die vielfältigen Ursachen gewaltsamer Konflikte und Auseinandersetzungen sind nicht nur eine Herausforderung für die Friedensforschung, sie bringen auch neue Anforderungen für das nationale und internationale politische Handeln mit sich. Zivile Antworten auf diese Herausforderungen lassen sich nicht mehr allein mit den Mitteln der traditionellen Diplo- 
matie bewerkstelligen. Vielmehr müssen wir auch die Instrumente der Entwicklungspolitik, der Umwelt-, Bildungs- oder Wirtschaftspolitik gezielt nutzen. Erfolgreiches Handeln setzt übergreifende Strategien voraus, die nicht an traditionellen Ressortgrenzen und Zuständigkeitsbereichen scheitern dürfen.

III.

Die damalige rot-grüne Bundesregierung hatte auf die veränderte Sicherheitslage bereits vor mehr als einem Jahrzehnt mit einem ersten Gesamtkonzept und einem Aktionsplan „Zivile Krisenprävention, Konfliktlösung und Friedenskonsolidierung" reagiert. Ausgangspunkt war ein erweiterter Sicherheitsbegriff, der politische, ökonomische, ökologische und soziale Stabilität umfasste. Insgesamt ging es darum, die Bedeutung einer primär auf friedliche Mittel und Ressourcen setzenden deutschen Außenpolitik zu unterstreichen.

Eine Vielzahl wichtiger Maßnahmen konnte entwickelt, entscheidende Impulse gegeben werden. Deutschland spielt inzwischen bei der Entwicklung und Verbreitung ziviler Maßnahmen eine wichtige und anerkannte Rolle. Insbesondere beim Aufbau einer Infrastruktur für die zivile Krisenprävention und Konfliktbearbeitung gehörte Deutschland damit zu den Vorreitern. Dafür stehen vor allem das Zentrum für Internationale Friedenseinsätze (ZIF), der Zivile Friedensdienst (ZFD), die krisenpräventive Ausrichtung der Entwicklungszusammenarbeit, die Förderung zivilgesellschaftlicher Projekte durch das Programm „zivik“, die Gruppe Friedensentwicklung (FriEnt) und die Gründung der Deutschen Stiftung Friedensforschung (DSF).

Leider hat die liberal-konservative Bundesregierung diese Ansätze nicht weiterentwickelt. Vor allem hat sie es unterlassen, aufbauend auf diesen Grundlagen eine weiterführende Gesamtstrategie deutscher Friedens- und Sicherheitspolitik zu entwickeln. Wir brauchen aber eine solche Strategie, wenn wir den Frieden weltweit nachhaltig und solidarisch fördern wollen.

Eine so geartete Strategie muss Grundlagen, Ziele und Handlungsfelder benennen und entsprechende Ressourcen bereitstellen, um damit einen verlässlichen Orientierungsrahmen für die deutschen und internationalen Partner zu bieten. Vor dem Hintergrund sich verändernder Formen von Konflikten und den tieferliegenden Ursachen darf eine solche Strategie nicht nur die strategische Ausrichtung des Auswärtigen Amtes beschreiben, sondern muss zu einer Strategie der gesamten deutschen Bundesregierung gemacht werden.

Im Kern kommt es bei der Formulierung einer solchen Strategie darauf an, den Stellenwert, die Ausrichtung und die Prioritäten für Deutschland neu zu bestimmen, um davon ausgehend konkrete Handlungsfelder, Maßnahmen und Projekte abzuleiten. Bisher ist völlig offen, welche Rolle Deutschland als politischer Akteur einnehmen will. Es fehlt an einer klaren Vision und einer Definition deutscher Interessen in und an Friedensoperationen.

Interessenpolitik in diesem Sinne heißt nicht zu definieren, in welchem Land wir welche wirtschaftlichen Zielsetzungen erreichen können oder wollen. Es geht vielmehr darum, klare
Prioritäten zu benennen, die sich aus unseren Fähigkeiten und Kompetenzen und vielleicht auch aus unserer Verantwortung ableiten lassen. Natürlich müssen wir auf aktuelle Krisen reagieren, aber wir müssen auch Themen und Regionen aufgreifen, an denen wir langfristig arbeiten und konzeptionelle, intellektuelle und finanzielle Energie investieren wollen.

\section{IV.}

Lassen Sie mich einige zentrale Handlungsfelder benennen, die aus meiner Sicht eine solche Strategie umfassen sollte:

1. Multilaterale Institutionen: Deutsches Engagement kann dazu beitragen, multinationale Institutionen handlungsfähiger zu machen und umgekehrt können diese das bilaterale deutsche Engagement verstärken. Sie sollten deshalb noch intensiver genutzt und mit einer strategischen Personalpolitik unterstützt werden. Es gilt den Ruf eines „ehrlichen Verhandlers“, der nicht vor allem eigennützige Ziele verfolgt, sondern sich dem Ziel Frieden verpflichtet fühlt, zu wahren.

2. Umfassender Politikansatz: Für eine stringente Friedensförderung und Konflikttransformation sind ein umfassender Politikansatz und das abgestimmte und vernetzte Handeln aller relevanten Akteure unabdingbar. Gerade die deutsche Ausprägung des Ressortprinzips steht dem immer wieder entgegen. Die federführende Rolle des Auswärtigen Amtes in diesem Bereich bedarf dringend einer Neujustierung.

3. Personalentwicklung und Kompetenzaufbau: Noch stärker als bisher müssen in Institutionen und Ministerien systematisch Kompetenzen im Bereich der Friedensförderung aufgebaut werden. Im Rahmen einer strategischen Personalplanung müssen die Vorzüge von Generalisten- und Rotationsprinzip mit der spezifischen Profilierung im Bereich der Konfliktbearbeitung kombiniert werden.

4. Stärkung der Strukturen vor Ort: Vor allem in Konfliktregionen ist eine Stärkung der Strukturen vor Ort erforderlich, in den deutschen Botschaften, aber auch in der systematischen Vernetzung mit Friedensoperationen und entwicklungspolitischen Projekten. Darüber hinaus ist ein „local ownership“ zentral für die Nachhaltigkeit der Friedensförderung.

5. Zivilgesellschaft: Eine erfolgreiche Strategie, auch wenn sie primär staatliche Akteure in den Blick nimmt, braucht die Unterstützung der Zivilgesellschaft. Sie sollte deshalb in enger Kooperation mit ihr und mit unabhängigen Experten entwickelt werden. Wichtig ist es, darauf $\mathrm{zu}$ achten, dass gerade deren Autonomie den Mehrwert zivilgesellschaftlichen Engagements erst ermöglicht.

6. Ressourcen: Die Ausstattung mit ausreichenden finanziellen und personellen Ressourcen ist zentral für die Umsetzung einer ambitionierten Strategie. Dazu braucht es einer klaren, auch haushaltspolitischen Prioritätensetzung, um das Ungleichgewicht zwischen zivilen und militärischen Maßnahmen zu überwinden.

7. Unterstiutzung: Um öffentliche und politische Unterstützung zu mobilisieren, müssen wir mehr Anstrengungen auf die Kommunikation von Zielen und Inhalten verwenden. 
Gleichzeitig sollten die Erwartungen an unser Engagement auch unseren Möglichkeiten entsprechen.

Diese Handlungsfelder sind aber allenfalls erste Eckpunkte für einen notwendigen Strategiebildungsprozess. In einem solchen Prozess muss es darum gehen, eine präzisere und transparentere Bestimmung von Interessen, Zielen und Schwerpunkten vorzunehmen. Dabei müssen alle relevanten staatlichen - zivilen und militärischen - Akteure ebenso eingebunden werden wie die Vertreter der Zivilgesellschaft. In diesem Sinne wäre ich auch für Kritik und weitergehende Vorschläge von Ihrer Seite dankbar.

\section{V.}

Seit den 1990er Jahren ist das internationale Krisenmanagement nicht nur von einem erweiterten Aufgabenspektrum geprägt, sondern auch von längeren Zeithorizonten und einer Zunahme der Akteure - lokalen, nationalen und internationalen, staatlichen und nichtstaatlichen. Ein abgestimmtes und vernetztes Handeln wird somit immer wichtiger. Die Debatten dazu finden in Deutschland jedoch mit Blick auf das vermeintliche Konzept der ,Vernetzten Sicherheit' zunehmend ideologisiert statt. Dies führt dazu, dass die Auseinandersetzung darüber, wie Krisenprävention, Konfliktbearbeitung und Friedenskonsolidierung gemeinsam erfolgreich gelingen können, in den Hintergrund rückt.

Es ist gerade die Heterogenität der Akteure, die ein abgestimmtes Vorgehen notwendig macht, die unterschiedlichen Denk- und Handlungsansätze zusammenbringt und eine Verständigung auf gemeinsame Ziele fördert. Dies ist Herausforderung und Chance gleichermaßen. Dabei darf ein solches abgestimmtes Handeln nicht die unterschiedlichen Lösungsansätze und Vorgehensweisen ersticken. Es sollte vielmehr unnötige Konkurrenzen ausschließen und zu einem abgestimmten Auftreten und Handeln in Krisen- und Konfliktregionen führen.

Ein zentrales Element für ein abgestimmtes und vernetztes Handeln ist eine Verständigung zumindest auf ein Bündel gemeinsamer Ziele, die sich als Zielfamilien darstellen lassen und innerhalb derer sich die Akteure wiederfinden können. Solche Zielfamilien könnten aus meiner Sicht die notwendige Orientierung und Motivation für ein vernetztes Handeln stiften, damit die wichtigsten Akteure an letztlich einem Strang ziehen. Wirksames Handeln hängt schließlich davon ab, dass die spezifischen Fähigkeiten verschiedener Akteure bestmöglich zur Geltung kommen, sich sinnvoll ergänzen und nicht duplizieren oder gar behindern.

Dies erfordert ein „Management of Diversity“, bereits in einem frühestmöglichen Planungsstadium, ein „Management of Diversity“, das die unterschiedlichen Zielsetzungen und Interessen der handelnden Akteure wahrnimmt und davon ausgehend Synergien sucht. Dabei geht es aus meiner Sicht sowohl um die Optimierung der Zusammenarbeit auf staatlicher Ebene, wie auch die verstärkte Kooperation von staatlichen und nichtstaatlichen Organisationen miteinander und der zivilen Akteure untereinander.
Ich hoffe, dass es vor dem Hintergrund dieser Ansätze, die wir derzeit gerade im Unterausschuss „Zivile Krisenprävention und Vernetzte Sicherheit“ diskutieren, gelingt, das Miteinander der verschiedenen Akteure zu stärken und die Debatte auf eine neue konstruktive Ebene zu heben. Wichtig ist es dabei aus meiner Sicht, dass auch das Militär eine Rolle einnimmt, die primär die Anforderungen der zivilen Akteure im Blick hat und diese befördert.

\section{VI.}

Eine als zaudernd oder gar unberechenbar wahrgenommene Außen- und Sicherheitspolitik würde nicht nur das weltweite Ansehen Deutschlands als verlässlichem Partner mindern, sie wäre auch ein falsches Signal für die internationalen Anstrengungen zur Friedensförderung.

Für mich ist es dabei keine Frage, dass wir die Krisenprävention und Konfliktüberwindung mit zivilen Mitteln stärken und auf eine neue strategische Basis stellen müssen. Deutschland muss sein Engagement für Friedensförderung und Konflikttransformation ausbauen und inhaltlich weiterentwickeln. Damit dies mittelfristig auch gelingt, brauchen wir nicht nur politische und gesellschaftliche Unterstützung, sondern immer wieder auch wissenschaftliche Expertise.

Ich bin deshalb sehr dankbar, dass mit dem Studiengang „Master of Peace and Security Studies“ hier in Hamburg genau diese wissenschaftliche Expertise gefördert und bereitgestellt wird. Den Absolventinnen und Absolventen gratuliere ich recht herzlich zum erfolgreichen Abschluss des Studiums und würde mich sehr darüber freuen, die eine oder den anderen in der Politikberatung, in Ministerien oder internationalen Organisationen wiederzusehen. Ich wünsche Ihnen allen für Ihre weitere berufliche Zukunft viel Erfolg und auch persönlich alles erdenklich Gute. 